$00 \$ 03.00 / 0$

Adv. Complex Systems (2018) 1, 114

\title{
Trading behavior and excess volatility in toy markets
}

\author{
Matteo Marsili \\ Istituto Nazionale per la Fisica della Materia (INFM) \\ Trieste-SISSA Unit, \\ V. Beirut 2-4, Trieste I-34014 \\ Damien Challet \\ Institut de Physique Théorique, Université de Fribourg, \\ Perolles, CH-1700
}

\begin{abstract}
$A B S T R A C T$. We study the relation between the trading behavior of agents and volatility in toy markets of adaptive inductively rational agents. We show that excess volatility, in such simplified markets, arises as a consequence of $i$ ) the neglect of market impact implicit in price taking behavior and of ii) excessive reactivity of agents. These issues are dealt with in detail in the simple case without public information. We also derive, for the general case, the critical learning rate above which trading behavior leads to turbulent dynamics of the market.

KEYWORDS: statistical mechanics, El Farol bar problem, minority games, market impact, adaptive learning.
\end{abstract}

\section{Introduction}

The El Farol bar problem (Arthur, 1994) and its later refinement, the Minority Game (MG) (Challet and Zhang, 1997), where devised to study the competitive interaction of boundedly rational agents in socio-economic contexts. In the El Farol bar problem agents face the binary choice of either attending or not a bar which is enjoyable only if it is not too crowded. Arthur (1994) showed that inductive rationality is enough for the average attendance to self-organize around the comfort capacity of the bar.

The El Farol bar context is simplified even further in the Minority Game. Again agents compete in forecasting what the majority will do, in order to be on the opposite side, which is the rewarding one. Agents, like speculators in 
a financial market (Zhang, 1998), try to exploit predictability of the "market". This speculative pressure drives the system in a self-organized state where, on average, half of the agents take each of the two possible actions at each time.

This confirms the naive expectation that speculative trading eliminates arbitrages - i.e. predictable patterns - in markets. In addition, the MG allows to focus attention on the fluctuations around the average and allows to address the question of whether and to which extent adaptive agents reduce or amplify fluctuations. Fluctuations in the MG are directly related to the profit losses and hence to wastes of resources. This is reminiscent of volatility in financial markets, which is an undesirable consequence of speculative trading.

The MG describes in a nutshell the interplay between predictability and volatility in a market with speculative trading. Since it allows to quantify both predictability $H$ and volatility $\sigma^{2}$ (see below), the MG allows to address questions of both information efficiency (predictability) and market stochasticity (volatility).

Much numerical work Challet and Zhang, 1997; Savit et al., 1999; Johnson et al., 1998; Cavagna, 1999; Challet and Marsili, 1999; Cavagna et al., 1999) as well as approximate analytical approaches (Zhang, 1998; Challet and Zhang, 1998; Johnson et al., 1999) have been devoted to the understanding of the MGi has culminated in the exact solution (Challet et al., 2000A; Marsili et al., 2000) in the limit of infinitely many agents, thanks to statistical mechanics tools of disordered systems (Mezard et al., 1987). The emerging picture is that the speculative trading of agents actually minimizes market's predictability. As the number of agents increases, predictability is reduced and beyond a critical number of agents, the market becomes unpredictable (to agents). At the same time also the volatility of the market decreases. Beyond the critical number, however, the market remains unpredictable but volatility starts increasing. In addition excess volatility due to crowd effects arise, which manifest in an anti-persistent behavior of the market (Savit et al., 1999; Challet and Marsili, 1999).

If the competitive aspects of the MG are related to predictability, from the point of view of volatility the MG is similar in nature to coordination games (Myerson, 1997). Indeed among all possible ways to exploit market's predictability, each agent has incentives to favor those which reduce market's volatility. Since the MG is a closed dynamical system, fluctuations inevitably depend on the behavior of agents.

Here we study in detail the relation between volatility and the behavior of agents. There are two key aspects of agent behavior which have been found to dramatically affect volatility in the MG:

1 The market impact and the extent to which agents account for it. In the original MG, agents neglect their market impact and behave as price takersf. This behavior minimizes market's predictability but this leads to large

$\dagger$ A collection of references on the Minority Game is available at the site http://www .unifr.ch/econophysics/minority.

$\ddagger$ Price taking behavior means that, when an agent considers counter-factually the results of the various choice he/she could have made, he/she does not account for the fact that the market "price" depends on the choice he/she actually made. If he/she had taken a different decision the "price" also would have changed. In few words, one may say that agents, rather 
volatility increases (crowd effects) when the market is crowded. This excess volatility is dramatically reduced as soon as agents start accounting, even approximately, for their impact. When agents behave strategically, properly accounting for their impact on the market, volatility is reduced to zero.

2 Agents reactivity, i.e. how readily and strongly do agents react to the outcome of the game. Fast learning rates may dramatically enhance volatility in the MG, with respect to that with small learning rates.

These results were found recently for the MG in its full complexity Challet et al., 2000A; Marsili et al., 2000) and for a modified version of it Cavagna et al., 1999) 1. Here we derive these result in the simplest case where there is no public information. This case allows for a quite simple treatment where the result is not obscured by the technicalities needed to deal with the MG with information.

In addition it allows to understand the origin of enhanced volatility for fast learning rates. This arises as a transition from a smooth dynamics to a more complex dynamical behavior which is reminiscent of chaotic or turbulent systems.

Then we move to the MG with information and briefly review the results of Marsili et al. (2000). In addition we derive a new result for the critical learning rate, as a function of $\alpha$ above which crowd effects arise in the MG.

We close with some discussion of the results.

\section{The model without information}

The MG is a toy model of a market under the simplifying assumption that the market is a repeated game of $N$ interacting agents. In real markets, agents can decide not to participate. In addition real agents trade at different frequencies. In the $\mathrm{MG}$ all agents have to trade at each time step. If $a_{i}(t)$ represents the investment of agent $i$ at time $t$, his/her profit from this transaction is

$$
u_{i}(t)=a_{i}(t) R[A(t) / N], \quad A(t)=\sum_{j=1}^{N} a_{j}(t) .
$$

The return $R(A / N)$ depends on the aggregate investment $A(t)$. This functional dependence embodies the market mechanism and it must be such that agents cannot extract a positive profit from just trading. Hence the game is a negative sum game:

$$
\sum_{i=1}^{N} u_{i}(t)=A(t) R[A(t) / N] \leq 0, \quad \forall t .
$$

In addition we further assume that $a_{i}(t)$ can take only two values \pm 1 . Hence agents choosing the minority sign $a_{i}=-\operatorname{sign} A$ gain a profit $|R(A / N)|$ whereas

than playing against other rational agents, thinks as if they were playing against an exogenous price process.

$\dagger$ Challet et al. (2000C) showed that the model introduced by Cavagna et al. (1999) has exactly the same collective behavior of the original $\mathrm{MG}$ in the limit $N \rightarrow \infty$. 
the majority of agents loose an amount $-|R(A / N)|$. The simplest choice $R(x)=$ $-x$ will be made henceforth

Naively, each player faces the problem of anticipating what the majority will do, in order to do the opposite. This is similar in essence to the El Farol bar problem (Arthur, 1994) and to many other situations where players have to share a limited resource.

The last ingredient of the MG is public information. Since the effects which we wish to focus on emerge even in the absence of public information, we shall introduce this ingredient later on.

Let us generalize the model and allow agents to behave in a stochastic way. Hence we introduce the probabilities $\pi_{i}$ with which agent $i$ plays action $a_{i}=+1$ (with $\operatorname{Prob}\left\{a_{i}=-1\right\}=1-\pi_{i}$ ). With $\pi_{i}=0$ or 1 we recover the deterministic case, whereas $\pi_{i}=1 / 2$ means that agent $i$ relies on the toss of a fair coin, to decide his/her action. Expected values over $\pi_{i}$ are denoted by $\langle\ldots\rangle$.

For any state $\vec{\pi}=\left(\pi_{1}, \ldots, \pi_{N}\right)$, we define

$$
H(\vec{\pi})=\langle A\rangle^{2}, \quad\langle A\rangle=\sum_{i=1}^{N}\left(2 \pi_{i}-1\right)
$$

and

$$
\sigma^{2}(\vec{\pi}) \equiv\left\langle A^{2}\right\rangle=H+4 \sum_{i=1}^{N} \pi_{i}\left(1-\pi_{i}\right) .
$$

$H$ measures information efficiency. Indeed $H>0$ implies $\langle A\rangle \neq 0$ which means that one can predict the minority action $-\operatorname{sign}\langle A\rangle$. When $H=0$ the outcome $A$ is unpredictable. In both cases, $\sigma^{2}$ is simply related to the total loss of agents $\sigma^{2}=-N \sum_{i}\left\langle u_{i}\right\rangle$ and hence it measures optimality. Volatility can be defined as the excess fluctuations $\sigma^{2}-H$ of the market.

\section{Nash equilibria}

The key concept to analyze the outcome of strategic interactions are the Nash equilibria of game theory (Myerson, 1997). The MG is an $n$-players game, where each agent $i$ may play pure strategies $a_{i}=+1$ or $-1-$ or mixed strategies $\pi_{i}-$ and the payoff matrix is specified by Eq. (2.1). Nash equilibria (Myerson, 1997) are defined as those states $\vec{\pi}$ in which agents have no payoff incentive to change their behavior.

The purpose of this section is to provide a reference framework for our later discussion on adaptive agents. More precisely it is of interest to undestand under what conditions adaptive learning can lead to the outcome one would expect from rational players, i.e. to a Nash equilibrium. Hence we shall limit our discussion to a rather elementary levell.

Let us consider a state $\vec{\pi}^{(j)}$ where $N-2 j$ agents play mixed strategies $\pi_{i}=1 / 2$

$\ddagger$ This is justified a posteriori by power expansion of $R(x)$ in $x$ because $A(t)$ is typically of order $\sqrt{N}$. The original choice in (Challet and Zhang, 1997; Savit et al. 1999) had $R(x)=\operatorname{sign} x$ which can also be dealt with similarly (ee appendix of Challet et al., 2000B).

$\dagger$ In what follows, Nash equilibria are vectors $\vec{\pi}$ such that no agent can increase his/her payoff by changing his/her mixes strategy $\pi_{i}$, if others stick to theirs. 
and $2 j$ play pure strategies. The frozen agents playing pure strategies are divided into two groups of $j$ agents playing opposite actions. This is a Nash equilibrium.

In order to show this, we observe that the $2 j$ agents playing pure strategies have no incentive to change it, because if they deviate from their strategy they suffer a loss. Agents playing mixed strategies must, on the other hand be indifferent between the two action, which occurs if $\left\langle A-a_{i}\right\rangle=\sum_{j \neq i}\left(2 \pi_{j}-1\right)=0$.

Overall, by simple combinatorics we find that there are

$$
\Omega(N)=\sum_{j=0}^{[N / 2]} 2\left(\begin{array}{c}
N \\
N-2 j
\end{array}\right)\left(\begin{array}{c}
2 j \\
j
\end{array}\right)-1
$$

Nash equilibria of this form (here $[N / 2]$ is the integer part of $[N / 2]$ ). For $N$ even, the information efficiency and optimality of these equilibria are quantified by

$$
H\left(\vec{\pi}^{(j)}\right)=0, \quad \sigma^{2}\left(\vec{\pi}^{(j)}\right)=N-2 j .
$$

This makes it clear that agents playing mixed strategies $\pi_{i}^{(j)}=1 / 2$ are those who contribute to the value of $\sigma^{2}$. Hence they are responsible for inefficiencies (excess volatility). As the number $j$ of frozen agent pairs increases the equilibrium becomes more and more efficient $\left\lceil\right.$ and $\sigma^{2}$ decreases. The equilibria $\vec{\pi}^{([N / 2])}$, where agents attain the minimal value of $\sigma^{2}$, shall be called optimal henceforth. At the other extreme, the symmetric Nash equilibrium in which all agents play mixed strategies $\left(\pi_{i}^{(0)}=1 / 2\right.$ for all $\left.i\right)$ is the most inefficient.

The number of frozen agents can increase if we allow for coordination devices such as binding agreements or public information. If agents $i$ and $k$, playing $\left(\pi_{i}, \pi_{k}\right)=(1 / 2,1 / 2)$ coordinate their actions choosing to play either $\left(\pi_{i}, \pi_{k}\right)=$ $(0,1)$ or $\left(\pi_{i}, \pi_{k}\right)=(1,0)$ with some probability, their payoffs increase (which increases the payoffs of other agents as well). By this, however, we are assuming

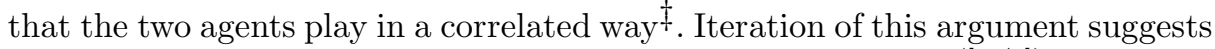
that agents will naturally choose one of the optimal equilibria $\vec{\pi}^{([N / 2])}$ if they can correlate their play.

Summarizing, the single stage game has an huge number of Nash equilibria for $N \gg 1$ (Eq. 3.1). This raises the problem of equilibrium selection: Which of these equilibria describes the behavior of deductive rational agents? Unfortunately game theory, on the basis of rationality and common knowledge alone, does not provide an unambiguous answer to this question.

Since Nash equilbria are very different in nature, with values of $\sigma^{2}$ ranging from 0 to $N$, this means that, in fact, we cannot draw any conclusion at all. Hence, as suggested by Arthur (1994), one has to abandon deductive rationality and replace game-theoretic players by inductively rational adaptive agents.

\footnotetext{
$\dagger$ In game theoretical terms one may say that for any Nash equilibrium $\pi^{(j)}$ there is an equilibrium $\pi^{(j+1)}$ which is Pareto dominant: In the latter each agent is not worse off than in the first and there is at least one agent which is strictly better off.

$\ddagger$ We refer the reader to Myerson, 1997) for a detailed discussion of coordination games and correlated equilibria.
} 


\section{Adaptive agents}

Agents are adaptive, in the sense that they learn from past experience which choice is the best one. The past experience of agent $i$ is stored in $\Delta_{i}(t)$ which is related to the cumulated payoff of the two possible actions. $\Delta_{i}(t)>0$ means that the action $a_{i}=+1$ is (perceived as) more successful than $a_{i}=-1$ and vice-versa. On the basis of their experience $\Delta_{i}(t)$ up to time $t$, the behavior of agents is described by

$$
\operatorname{Prob}\left\{a_{i}(t)= \pm 1\right\} \equiv \frac{1 \pm m_{i}(t)}{2}, \quad \text { with } \quad m_{i}(t)=\chi_{i}\left[\Delta_{i}(t)\right]
$$

The function $\chi_{i}(x)$ is an increasing function of $x$ with $\lim _{x \rightarrow \pm \infty} \chi_{i}(x)= \pm 1$. Hence the probability with which agent $i$ plays action $a_{i}= \pm 1$ increases with $\Delta_{i}(t)$.

The connection with mixed strategies is $m_{i}=2 \pi_{i}-1$. Here we choose a different notation to stress the fact that $m_{i}(t)$ is not a mixed strategy, but rather it encodes a behavioral rule. Note that $m_{i}=\left\langle a_{i}\right\rangle$.

The way in which $\Delta_{i}(t)$ is updated is the last element of the learning dynamics. We assume that

$$
\Delta_{i}(t+1)=\Delta_{i}(t)-\frac{1}{N}\left[A(t)-\eta_{i} a_{i}(t)\right]
$$

The idea is: if agent $i$ observes $A(t)<0$ he/she will generally increase $\Delta_{i}$ and hence his/her probability of playing $a_{i}=+1$ at the next time step. The $\eta_{i}$ term above describe the fact that agent $i$ may account for his/her own contribution to $A(t)$. For $\eta_{i}=1$ indeed, agent $i$ considers only the behavior of other agents $A(t)-a_{i}(t)$ and does not react to his/her own action $a_{i}(t)$.

The question of interest is: Do agents converge to a Nash equilibrium under this dynamics? If yes, to which equilibrium do agents converge?

\subsection{NAIVe Agents $\eta_{i}=0$}

In the MG agents are naive and do not account for their impact on the aggregate, i.e. $\eta_{i}=0$.

Let us focus on the long time dynamics for $N \gg 1$. In particular we follow (Marsili et al., 2000) and introduce a time variable $\tau=t / N$. A superscript ${ }^{(c)}$ denotes variables in the continuum time (e.g. $\left.\Delta_{i}^{(c)}(\tau)=\Delta_{i}(N \tau)\right)$. Then

$$
\Delta_{i}^{(c)}(\tau+d \tau)=\Delta_{i}^{(c)}(\tau)-\frac{1}{N} \sum_{t=N \tau}^{N(\tau+d \tau)} A(t) .
$$

For $N \gg 1$ we can use the law of large numbers and conclude that the last term is well approximated by $-d \tau\langle A\rangle_{\tau}$. Here the subscript $\tau$ implies that the average is taken over the probabilities $\left[1 \pm m_{i}^{(c)}(\tau)\right] / 2$ of the actions $a_{i}= \pm 1$, and we are assuming that $\chi_{i}(x)$ is smooth enough (see later) so that these probabilities are approximately constant over the time interval $[\tau, \tau+d \tau)$. Hence we find that

$$
\dot{\Delta}_{i}^{(c)}(\tau)=-\langle A\rangle_{\tau}=-\sum_{i=1}^{N} m_{i}^{(c)}(\tau) .
$$


It is quite easy to find that $H$ is minimized along the trajectories of the learning dynamics with $\eta_{i}=0$ for all $i$.

Indeed, with the notation $\langle\ldots\rangle_{\tau}$ for averages taken at time $\tau$

$$
\dot{H}=2\langle A\rangle_{\tau} \sum_{i=1}^{N} \frac{\partial\langle A\rangle_{\tau}}{\partial m_{i}^{(c)}} \dot{m}_{i}^{(c)}=-2 H \sum_{i=1}^{N} \chi_{i}^{\prime}\left(\Delta_{i}^{(c)}\right)
$$

where we have assumed that $\left\langle\chi_{i}\left(\Delta_{i}\right)\right\rangle_{\tau} \simeq \chi_{i}\left(\Delta_{i}^{(c)}\right)$, which is correct to leading order in $1 / N$.

Since $\chi_{i}^{\prime}(x) \geq 0$ for all $x$ and $i$, we conclude that $\dot{H} \leq 0$ i.e. naive agents minimize the predictability $H$, and $H \rightarrow 0$ as $t \rightarrow \infty$. There are many states with $H=0$ and the dynamics select that which is "closer" to the initial condition. To be more precise, let $\Delta_{i}(0)$ be the initial condition (which encodes the prior beliefs of agents on which action is best). Then, as $\tau \rightarrow \infty,\langle A\rangle_{\tau} \rightarrow 0$ and $\Delta_{i}^{(c)}(\tau)$ converges to

$$
\Delta_{i}^{(c)}(\infty)=\Delta_{i}(0)+\delta A, \quad \text { with } \quad \delta A=\int_{0}^{\infty} d \tau\langle A\rangle_{\tau} .
$$

The condition $\langle A\rangle_{\infty}=0$ provides an equation for $\delta A$

$$
0=\sum_{i=1}^{N} \chi_{i}\left(\Delta_{i}(0)+\delta A\right) .
$$

By the monotonicity property of $\chi_{i}$, this equation has one and only one solution.

The asymptotic state of this dynamics is information-efficient $(H=0)$, but it is not optimal. Indeed, in general, this state is not a Nash equilibrium. Typically we find $\sigma^{2} \propto N$. Only in the special case $\Delta_{i}(0)=0$ and $\chi_{i}(0)=0$ for all $i$, we recover the symmetric Nash equilibrium $\vec{\pi}^{(0)}=(1 / 2, \ldots, 1 / 2)$ where $\sigma^{2}=N$.

\subsection{LESS NAIVE AGENTS $\eta_{i}>0$}

It is easy to check that with $\eta_{i}>0$, following the same steps as in the previous section, the learning dynamics of agents minimize the function

$$
H_{\eta}=\langle A\rangle^{2}-\sum_{i=1}^{N} \eta_{i} m_{i}^{2} .
$$

Because of the new term, $H_{\eta}$ attains its minima on the boundary of the domain $[-1,1]^{N}$. In other words, $m_{i}= \pm 1$ for all $i$ which means that agents play pure strategies $a_{i}=m_{i}$. The stable states are optimal Nash equilibria for $N$ even. By playing pure strategies agents minimize the second term of $H_{\eta}$. Of all corner states where $m_{i}^{2}=1$ for all $i$, agents select those with $\langle A\rangle=0$ by dividing into two equal groups playing opposite actions. All these states have minimal "energy" $H_{\eta}=-\sum_{i} \eta_{i}$. Which of these states is selected depends on the initial conditions $\Delta_{i}(0)$.

Note that the set of stable states is disconnected. Each state has its basin of attraction in the space of $\Delta_{i}(0)$. The stable state changes discontinuously as $\Delta_{i}(0)$ is varied. This contrasts with the case $\eta_{i}=0$ where Eq. (4.7) implies that 
the stationary state changes continuously with $\Delta_{i}(0)$ and the set of stationary states is connected.

For $N$ odd, similar conclusions can be found. This can be understood by adding a further agent to a state with $N-1$ (even) agents in a Nash equilibrium. Then $H_{\eta}=\left(1-\eta_{N}\right) m_{N}^{2}$, so for $\eta_{N}<1$ the new agent will play a mixed strategy $m_{i}=0$, whereas for $\eta_{N}>1$ it will play a pure strategy. In both cases other agents have no incentive to change their position. In this case we find $\sigma^{2} \leq 1$.

Summarizing, when agents account for their impact on the aggregate, they attain not only an information-efficient state, but also a optimal Nash equilibrium with $\sigma^{2}=H=0$.

It is remarkable how the addition of the parameter $\eta_{i}$ radically changes the nature of the stationary state. Most strikingly, fluctuations are reduced by a factor $N$.

\subsection{NAIVE AGEnts LEARning AT A FAST RATE}

The difference between the behavior of naive and non naive agents becomes even stronger if agents are naive $\left(\eta_{i}=0\right)$ and very reactive. In order to quantify more precisely what we mean, let us assume thati

$$
\chi_{i}(\Delta)=\tanh (\Gamma \Delta), \quad \forall i .
$$

Here $\Gamma$ is the learning rate, which measures the scale of the reaction in agent's behavior (i.e. in $m_{i}$ ) to a change in $\Delta_{i}$. We also assume that agents have no prior beliefs: $\Delta_{i}(0)=0$. Hence $\Delta_{i}(t) \equiv y(t) / \Gamma$ is the same for all agents. From the results discussed above, we expect, in this case the system to converge to the symmetric Nash equilibrium $m_{i}=0$ for all $i$. This is not going to be true if agents are too reactive, i.e. if $\Gamma>\Gamma_{c}$, as we shall see shortly.

Indeed $y(t)=\Gamma \Delta_{i}(t)$ satisfies the equation

$$
\begin{aligned}
y(t+1) & =y(t)-\frac{\Gamma}{N} \sum_{i=1}^{N} a_{i}(t) \\
& \simeq y(t)-\Gamma \tanh [y(t)]
\end{aligned}
$$

where the approximation in the last equation relies on the law of large numbers for $N \gg 1$. Eq. (4.10) is a dynamical system. The point $y^{0}=0$ is stationary, but it is easy to see that it is only stable for $\Gamma<\Gamma_{c}=2$. For $\Gamma>2$, a cycle of period 2 arises, as shown in figure 11. This has dramatic effects on the optimality of the system. Indeed, let $\pm y^{*}$ be the two values taken by $y(t)$ in this cycleff. Since $y(t+1)=-y(t)= \pm y^{*}$ we still have $\langle A\rangle=0$ and hence $H=0$. On the other hand $\sigma^{2}=N^{2} y^{* 2}$ is of order $N^{2}$, which is even worse than the symmetric Nash equilibrium where $\sigma^{2}=N$.

This transition from a state where $\sigma^{2} \propto N$ to a state with $\sigma^{2} \propto N^{2}$ is generic

$\dagger$ In this section we assume that agents follow a Logit model of discrete choice where the probability of choice $a$ is proportional to the exponential of the "score" $U_{a}$ of that choice: $\pi(a) \propto e^{\Gamma U_{a} / 2}$ With only two choices $a= \pm 1, \pi(a)=(1+a m) / 2$ and $\Delta=U_{+}-U_{-}$, we recover Eq. (4.9). This learning model has been introduced by Cavagna et al. (1999) in the context of the MG.

$\ddagger \pm y^{*}$ are the two non-zero solutions of $2 y=\Gamma \tanh (y)$. 


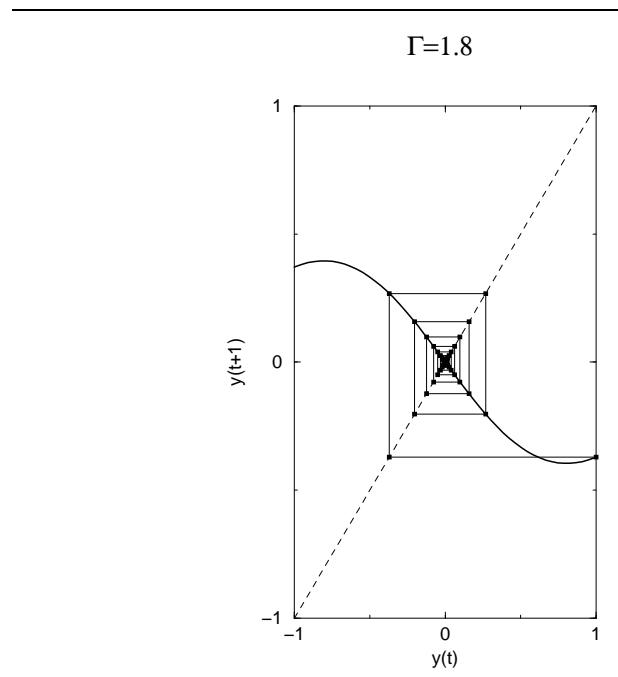

$\Gamma=2.5$

Figure 1: Graphical iteration of the map $y(t)$ for $\Gamma=1.8<\Gamma_{c}$ and $\Gamma=2.5>\Gamma_{c}$

in the minority game, as first observed by Savit et al. (1999). Much work has been done by Johnson et al. to describe this effect quantitatively in terms of "crowds" and "anti-crows". This approach is however static and does not reveal the dynamical nature of the transition, which was first discussed in Challet and Marsili, 1999). The transition from these two regimes as the learning rate $\Gamma$ is changed has been first observed in (Cavagna et al., 1999) for the MG.

We shall see below that the simple approach followed here can be generalized to the full minority game and it allows to derive the critical learning rate $\Gamma_{c}(\alpha)$ as a function of the parameter $\alpha$ of the MG.

\section{The MG with information}

The same qualitative behavior occurs when agents have access to a public information represented by an integer variable $\mu$ ranging from 1 to $P . \mu$ may either be related to past outcomes of the game (Challet and Zhang, 1997) or, as first suggested by Cavagna (1999), it may be just randomly and uniformly drawn from $\{1, \ldots, P\}$. The idea is that, agents resort to simple schemata or rules, which prescribe an action for each value of $\mu$. Each agent is initially endowed with $S$ such rules which are drawn at random among all possible $2^{P}$ binary functions. Agents take their choice on the basis of "scores" $U_{s, i}$ which they assign to each strategy $s$, and they update scores in a way which is similar to Eq. (4.2). Savit et al. (1999) has shown that the relevant variable is $\alpha=P / N$ : Intensive (i.e. $N$ independent) quantities, such as $\sigma^{2} / N$, display a behavior which does not depend on $P$ and $N$ separately, but only on their ratio $\alpha$. We refer the interested reader to (Marsili et al., 2000) for a discussion of the general case. Here we focus on the $S=2$ case, where following (Challet and Marsili, 1999; Challet et al., 2000A , we let $s= \pm 1$ be the label of the two possible rules of each agent and $s_{i}(t)$ be the choice actually taken by agent $i$ at time $t$. Following the notation of (Challet and Marsili, 1999; Challet et al., 2000A), the action 
taken by agent $i$ at time $t$, if he/she chooses to follow strategy $s_{i}(t)$ is $a_{i}(t)=$ $\omega_{i}^{\mu(t)}+s_{i}(t) \xi_{i}^{\mu(t)}$, where $\mu(t)$ is the value taken by public information 1 . The decision process of agent $i$ is hence encoded, as in Eq. (4.1) above, in the equation

$$
\operatorname{Prob}\left\{s_{i}(t)= \pm 1\right\} \equiv \frac{1 \pm m_{i}(t)}{2}, \quad \text { with } \quad m_{i}(t)=\chi_{i}\left(\Delta_{i}\right)
$$

Here $\Delta_{i}(t)$ is the difference between the scores of the two schemata, which are updated according to the analog of Eq. (4.2):

$$
\Delta_{i}(t+1)=\Delta_{i}(t)-\frac{A(t) \xi_{i}^{\mu(t)}-\eta_{i} \xi_{i}^{\mu(t)^{2}} s_{i}(t)}{N} .
$$

Refs. (Marsili et al., 2000; Challet et al., 20000) have shown that typical relaxation times in a system of $N$ agents are of order $N$. In order to have a meaningful dynamics in the limit $N \rightarrow \infty$ it is necessary to introduce a rescaled time $\tau=t / N$. This leads to a deterministic continuum time dynamics Challet and Marsili, 1999; Challet et al., 2000A which is independent of $N$. The validity of this description relies on the fact that typically $A(t) \sim \sqrt{N}$ and hence $\mid \Delta_{i}(t+$ 1) $-\Delta_{i}(t) \mid \sim 1 / \sqrt{N}$ is vanishingly small, which makes the continuum time approach exact as $N \rightarrow \infty$. As we shall see, this fails to be true if $\alpha<\alpha_{c}$ and agents are too reactive, since then $A(t) \sim N$.

Within the continuum time description one finds Challet et al., 2000A; Marsili et al., 2000) that with $\eta_{i}=0$ for all $i$, the behavior of agents again minimizes predictability $H$. It can be shown (Marsili et al., 2000) that the payoff of each agent $i$ increases with $\eta_{i}$ in the range $\eta_{i} \in[0,1]$. In addition, also global efficiency increases. If $P>\alpha_{c} N$, where $\alpha_{c}=0.3374 \ldots$, the improvement is smooth as a function of $\eta$. If, on the other hand, $P<\alpha_{c} N$ there is a sudden jump in $\sigma^{2}$ as soon as agents switch on a small parameter $\eta_{i}=\eta$. This is shown clearly in figure 2. This feature is captured qualitatively by the analytic calculation (in the replica symmetric ansatz) of (Challet et al., 2000A; Marsili et al., 2000), whose result is shown in Figure 3. For $\alpha=P / N<\alpha_{c}$, as the line $\eta=0$ is crossed from below, the system undergoes a first order phase transition with a discontinuous jump in $\sigma^{2}$. Note that for $\Gamma \gg \Gamma_{c}$ the jump in $\sigma^{2} / N$ is of more than two decades! This discontinuity arises because the nature of the stationary state changes abruptly: For $\eta \leq 0$ a finite fraction of agents play mixed strategies $\left(m_{i}^{2}<1\right)$ whereas for $\eta>0$ most of them play only pure strategies. For $\eta=1$ all agents play pure strategies and indeed the system converges to a Nash equilibrium

The transition for $\alpha>\alpha_{c}$ occurs at a value $\eta_{c}(\alpha)>0$ and it is smooth (second order). A deeper discussion on the nature of this transition and on its consequences may be found in (Marsili et al., 2000; De Martino et al., 2000).

\section{Learning rate in the MG for $\alpha<\alpha_{c}$}

As before, the performance of naive agents $\left(\eta_{i}=0 \forall i\right)$ may be much worse for $\alpha<\alpha_{c}$ if they are too reactive. This is shown numerically in figure 2. The effect

$\dagger \omega_{i}^{\mu}$ and $\xi_{i}^{m} u$ are such that $\omega_{i}^{\mu} \pm \xi_{i}^{m} u$ are two randomly choosen boolean functions of $\mu$ taking values in $\{ \pm 1\}$.

$\dagger$ Here Nash equilibria are defined with respect to the set of pure strategies $s_{i}= \pm 1$ which agents can take. See Marsili et al. (2000) for a detailed discussion. 


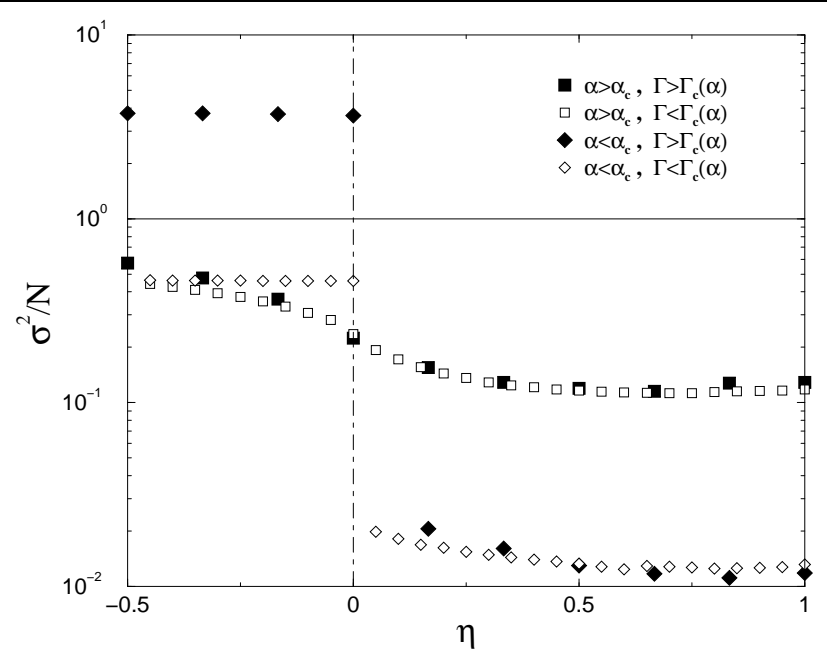

Figure 2: $\sigma^{2} / N$ as a function of $\eta$ for $S=2$ and $N=101$. Diamonds refer to $P=8\left(\alpha \simeq 0.079<\alpha_{c}\right)$ with $\Gamma=\infty$ (open symbol) and $\Gamma \ll \Gamma_{c}(\alpha)$ (full symbol). Squares refer to $P=64\left(\alpha \simeq 0.63>\alpha_{c}\right)$ with $\Gamma=\infty$ (open symbol) and $\Gamma \ll \Gamma_{c}(\alpha)$ (full symbol).

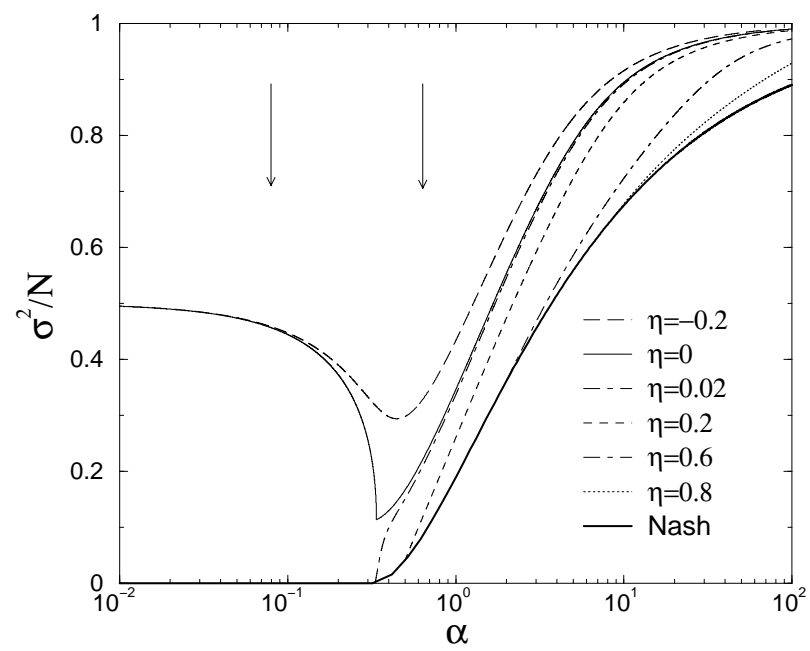

Figure 3: Approximate theoretical estimate of global efficiency $\sigma^{2} / N$ as a function of $\alpha$ for $S=2$ and several values of $\eta$ within the replica symmetric ansatz. The arrows mark the values of $\alpha$ to which Fig. 1 refers. 


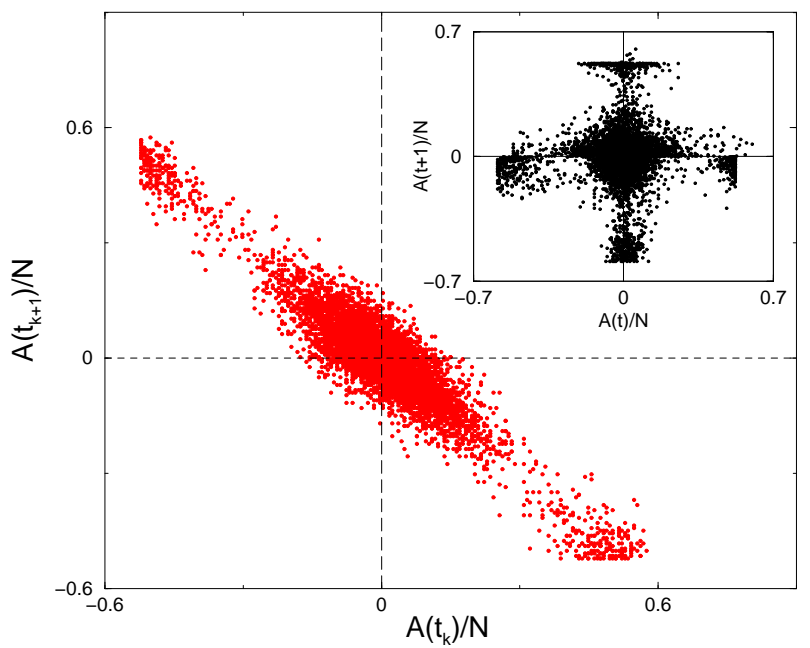

Figure 4: Plot of $A\left(t_{k+1}\right) / N$ vs $A\left(t_{k}\right) / N$ for the MG with $N=301$ agents, $\Gamma=\infty$ and $P=16(\alpha=0.053 \ldots)$. The sequence $t_{k}$ is such that the information is the same $\left(\mu\left(t_{k+1}\right)=\mu\left(t_{k}\right)\right)$. In the inset, the same plot with real time $t$.

is exactly the same as that discussed previously, in the absence of information $(P=1$ or $\alpha \simeq 0)$ : As the learning rate $\Gamma$ increases, the stationary solution $\Delta_{i}^{*}$ looses its stability and a bifurcation to a complex dynamics occurs. This is only possible in the low $\alpha$ phase, where the stationary state is degenerate and the system can attain $\left\langle A^{\mu}\right\rangle=0$ (intended as a time average) by hopping between different statesf. The plot of $A(t+1) / N$ vs $A(t) / N$, in figure 1 shows that indeed wild fluctuations occur in one time step: a finite fraction of agents change their mind at each time step. This is what causes, for fixed $P$, the cross over, first observed in (Savit et al., 1999), from the linear regime $\sigma^{2} \sim N$ to a quadratic dependence $\sigma^{2} \sim N^{2}$.

Clearly the continuum time limit, on which our analysis rests, breaks down because $A(t) \sim N$. Still one can compute the critical learning rate $\Gamma_{c}(\alpha)$ which marks the onset of complex dynamics. Let us focus attention on one value of $\mu=1$ and on the learning model of Eq. (4.9). Let us define the sequence of times $t_{k}$ such that $\mu\left(t_{k}\right)=1$ for the $k^{\text {th }}$ time. Fig. 1 shows that the dynamics of $A$ in this modified time is not smooth. We define $y_{i}(k)=\Gamma \Delta_{i}\left(t_{k}\right)$, which satisfies

$$
y_{i}(k+1)=y_{i}(k)-\frac{\Gamma}{N} \sum_{t=t_{k}}^{t_{k+1}-1} A(t) \xi_{i}^{\mu(t)}
$$

When $N \gg 1$, the sum involves $\sim P=\alpha N \gg 1$ terms and we may estimate it by the law of large numbers. Let $y_{i}^{*}$ be the stationary solution $\left(y_{i}(k+1)=\right.$ $\left.y_{i}(k)=y_{i}^{*}\right)$ of Eq. (6.1), then we can set $y_{i}(k)=y_{i}^{*}+\delta y_{i}(k)$ and study the linear

$\ddagger$ In the asymmetric phase $\alpha>\alpha_{c}$ the stationary state is unique and this effect is not possible. 


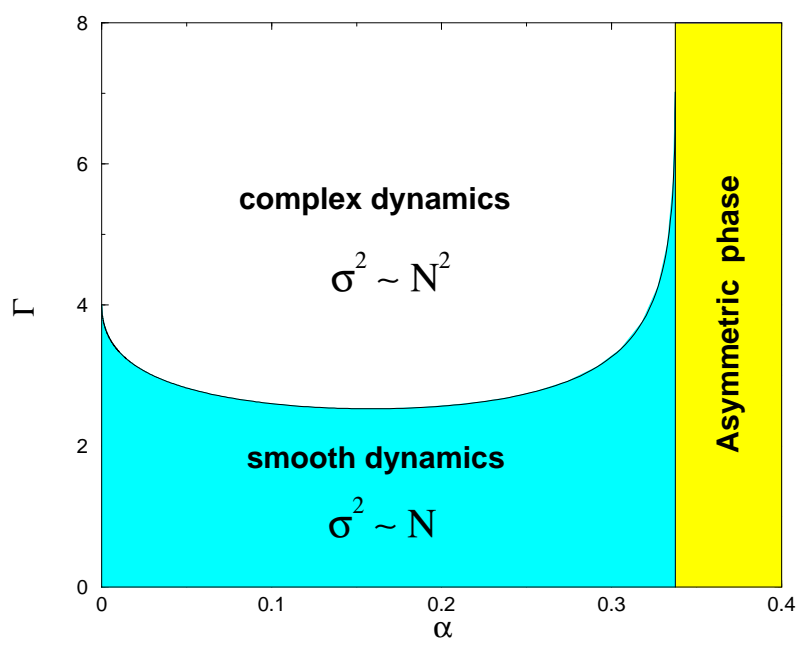

Figure 5: Phase diagram of the MG $\left(\eta_{i}=0\right)$ in the $(\alpha, \Gamma)$ plane.

stability of this solution. With the notation $\bar{R}=\sum_{\mu} R^{\mu} / P$, we find

$$
\delta y_{i}(k+1)=\sum_{j=1}^{N} T_{i, j} \delta y_{j}(k), \quad T_{i, j}=\delta_{i, j}-\alpha \Gamma \overline{\xi_{i} \xi_{j}}\left(1-m_{j}^{2}\right)
$$

where $m_{j}=\tanh \left(y_{j}^{*}\right)$. The solution $y_{i}^{*}$ is stable if the eigenvalues of $T_{i, j}$ are all smaller than 1 in absolute value. As $\Gamma$ increases, the smallest eigenvalue of $T_{i, j}$ becomes smaller that -1 . Thanks to the results of Sengupta and Mitra (1997), we have an analytic expression for this eigenvalue, which is $\lambda_{+}=1-\Gamma(1+$ $\sqrt{\alpha})^{2}(1-Q) / 2$. The stability condition $\lambda_{+}>-1$ then turns into

$$
\Gamma<\Gamma_{c}(\alpha) \equiv \frac{4}{[1-Q(\alpha)](1+\sqrt{\alpha})^{2}}, \quad Q(\alpha)=\frac{1}{N} \sum_{i=1}^{N} m_{i}^{2}
$$

which is our desired result. The function $Q(\alpha)$ is known exactly from the analytic solution (Challet et al., 2000A; Marsili et al., 2000). This yields a phase diagram in the $(\alpha, \Gamma)$ plane which is shown in figure 5. For $\alpha \rightarrow 0$ we find $\Gamma_{c} \rightarrow$ 地. As $\alpha \rightarrow \alpha_{c}, \Gamma_{c}$ converges to a finite value $(\simeq 7.0273 \ldots)$ with infinite slope. Numerical simulations suggests that, rather than a sharp transition, at $\Gamma_{c}(\alpha)$ the system undergoes a crossover between two distinct dynamical regimes. In the asymmetric phase $\left(\alpha>\alpha_{c}\right)$ the dynamics is always smooth, hence $\Gamma_{c}(\alpha)=\infty$.

The analysis of the stationary state for $\Gamma \gg \Gamma_{c}$ and the calculation of global quantities such as $\sigma^{2}$ is much more difficult than that in the smooth dynamical

$\dagger Q(\alpha)$ is given parametrically by $Q=1-e^{-z^{2}} /(\sqrt{\pi} z)-\left(z^{2}-1 / 2\right) \operatorname{erf}(z) / z^{2}$ and $\alpha=$ $\operatorname{erf}^{2}(z) /\left[2 z^{2}(1+Q)\right]$.

$\ddagger$ This differs from our previous result $\Gamma_{c}=2$ without information, because with $P=1$ in the MG half of the population has $a_{+, i}=a_{-, i}$ two equal strategies. This reduces by a factor 2 the effective number of adaptive agents, and accordingly $\Gamma_{c}$ takes a factor 2 . 
phase $\left(\Gamma \ll \Gamma_{c}\right)$. Johnson et al. (1999) devised an approximate scheme, which neglects the dynamical aspect of the problem, but gives expressions for $\sigma^{2}$ in good agreement with numerical data for $\alpha<\alpha_{c}$ and $\Gamma \gg \Gamma_{c}(\alpha)$. A microscopic, systematic derivation of $\sigma^{2}$ is a challenging problem, and we believe the dynamical system (6.1) is the starting point.

\section{Conclusion}

In its simplicity, the MG captures and reproduces a great deal of mechanisms, aspects and properties of real markets. In particular it provides a deep understanding of the relation between behavioral assumptions at the micro level and global macro behavior in a toy market. Here we have shown what properties of the behavior of agents are responsible for excess volatility in such toy markets. These are price taking behavior and excessive reactivity (fast learning).

This leaves us with the question of whether and to what extent these conclusions extend to real market contexts. Experimental studies in this direction promise to be very illuminating.

\section{References}

Arthur W. B., Inductive reasoning and bounded rationality, Am. Econ. Assoc. Papers and Proc 84, 406, 1994.

Cavagna A., Irrelevance of Memory in the Minority Game, Phys. Rev. E 59, R3783 (1999).

Cavagna A., J.P. Garrahan, I. Giardina, D. Sherrington, A thermal model for adaptive competition in a market, Phys. Rev. Lett. 83, 4429 (1999).

Challet D. and Zhang Y.-C., Emergence of cooperation and organization in an evolutionary game, Physica A 246, 407 (1997).

Challet D. and Zhang Y.-C., On the Minority Game : analytical and numerical studies, Physica A 256, 514 (1998).

Challet D. and M. Marsili, Phase Transition and Symmetry Breaking in the Minority Game, Phys. Rev. E 60, R6271 (1999).

Challet D., M. Marsili and R. Zecchina, Statistical Mechanics of Systems with Heterogeneous Agents: Minority games, Phys. Rev. Lett. 84, 1824 (2000A).

Challet D., M. Marsili and Y.-C. Zhang Modeling Market Mechanisms with Minority Games, Physica A 276, 284 (2000B).

Challet D., M. Marsili and R. Zecchina, e-print cond-mat/0004308.

De Martino A., M. Marsili and R. Zecchina forthcoming.

Johnson N.F. et al., Volatility and Agent Adaptability in a Self-Organizing Market, Physica A (258), 230 (1998).

Johnson N.F. et al., Crowds Effects and Volatility in a Competitive Market, Physica A 269, (1999).

Marsili M., D. Challet and R. Zecchina, Exact Solution of a modified El Farol's bar problem, Physica A (2000).

Mezard M., G. Parisi, M. A. Virasoro, Spin glass theory and beyond (World Scientific, 1987).

Myerson, R.B., Game Theory: analysis of conflict, Harvard Univ. Press (1997).

Savit R., Manuca R., and Riolo R., Adaptative competition, market efficiency and phase transition, Phys. Rev. Lett., 82, 2203 (1999).

Sengupta A. M., P. P. Mitra, Phys. Rev. E 60, 3389 (1999).

Zhang Y.-C., Evolving models of financial markets, Europhys. News 29, 51 (1998). 